\title{
Erratum to: $N$-Alkylation by Hydrogen Autotransfer Reactions
}

\author{
Xiantao $\mathrm{Ma}^{1,2} \cdot$ Chenliang $\mathrm{Su}^{2} \cdot$ Qing $\mathrm{Xu}^{1}$
}

(C) Springer International Publishing Switzerland 2016

\section{Erratum to: Top Curr Chem (Z) (2016) 374:27 DOI 10.1007/s41061-016-0027-1}

The original version of this article unfortunately contained a mistake.

The title of the article was incorrect. The correct title is " $N$-Alkylation by Hydrogen Autotransfer Reactions".

The original article was corrected.

The online version of the original article can be found under doi:10.1007/s41061-016-0027-1.

Qing Xu

qing-xu@wzu.edu.cn

1 College of Chemistry and Materials Engineering, Wenzhou University, Wenzhou, Zhejiang 325035, China

2 SZU-NUS Collaborative Innovation Center for Optoelectronic Science and Technology, Key Laboratory of Optoelectronic Devices and Systems of Ministry of Education and Guangdong Province, College of Optoelectronic Engineering, Shenzhen University, Shenzhen 518060, China 